\title{
Salud y participación
} social en Colombia. Análisis y reflexiones de las condiciones actuales para el ejercicio de la democracia

\section{César Alberto Pazo Fernández}

Administrador de Servicios de salud por la Universidad

Aprobado:

23 de enero de 2020 Recibido: 17 de julio de 2019 DOI: http://dx.doi.org/10.18566/rfts.v33n33.a05 de Antioquia; Magíster en Desarrollo por la Universidad Pontificia Bolivariana; Docente de la escuela de Ciencias de la Salud Universidad Nacional Abierta y a Distancia. E-mail.cesaralberto.pazo@gmail.com ORCID:00000003-2634-9246. 


\section{Resumen}

En el artículo son analizados los mecanismos de participación social en el sistema de salud colombiano. Las perspectivas de indagación son los contextos económico y político de la salud en Colombia, los escenarios legales de participación entre sociedad y empresas del sector salud, y finalmente, los aspectos que dificultan la adecuada relación entre los distintos actores. Como conclusión se propone que en un entorno en el que la salud es gestionada por actores privados, priman los intereses económicos particulares por encima del bienestar general, imposibilitando así la implementación de estrategias sanitarias pensadas desde las necesidades e intereses comunitarios.

Palabras clave

Sistema de salud, Participación social, Democracia.

\section{Abstract}

In this paper, the mechanisms of social participation in the Colombian health system are analyzed. The perspectives of inquiry are the economic and political context of health in Colombia, the legal settings of participation between society and private companies in the health sector, and finally the aspects that hinder the proper relationship between these different actors. The conclusion is that in an environment in which health is managed by private actors, private economic interests prevail over general societal well-being. Thus, private actors are shown to hinder the implementation of health strategies designed for the needs and interests of the community.

Key words

Health system, Social participation, Democracy. 


\section{Introducción}

Desde la puesta en vigencia del actual sistema de salud colombiano, las instituciones del Estado y el sector privado han puesto en marcha una serie de figuras organizativas que, en el marco de una sociedad democrática, garanticen a la ciudadanía su derecho a ser partícipes y gestores de las decisiones que en materia de salud beneficien el interés colectivo. Sin embargo, el mismo modelo económico bajo el cual se estructura el sistema de salud ha significado una barrera para la participación ciudadana, en donde la exclusión juega un papel fundamental en el logro de las metas en salud.

El presente artículo pretende sentar bases reflexivas en torno a la forma como está pensada la participación social en las instituciones de salud y por qué ha fracasado como un elemento democrático e incluyente. Para esto, serán abordados tres apartes que permitan comprender la problemática mencionada. En primer lugar, será descrito el contexto, que ofrece una mirada generalizada del sistema de salud, los modelos que lo cimientan y las necesidades de mercado que persigue. En segundo lugar, serán descritos los conceptos generales de participación social en salud, su importancia y los principales mecanismos en lo público y lo privado.

Por último, los aspectos críticos de la participación, abordando aspectos como el modelo económico, la estructura institucional, la captura del Estado por parte del sector privado encargado de la garantía del servicio y las tensiones entre empoderamiento y gobernanza.

\section{Contexto institucional}

En Colombia, las crisis económicas de Latinoamérica en la década de los ochenta y los lineamientos del Fondo Monetario Internacional para la reforma a los sistemas de salud, además de las directrices del Consenso de Washington, marcaron la pauta para la estructuración del actual sistema de salud. Es así como a partir de la Ley 100 de 1993, la salud se convirtió en un derecho exigible y defendible, soportado en principios como la universalidad, la igualdad y la solidaridad en el marco de un modelo de Estado Social de Derecho (Orozco, 2006, pp. 15-18). 
Dichos principios orientadores entran en discusión con el modelo económico neoliberal sobre el cual rige su estructura administrativa, debido a que el acceso a los servicios de salud pasa de ser una responsabilidad directa del Estado a ser prestados por una amplia amalgama de oferentes pertenecientes al sector privado. Así, surge un sistema de aseguramiento direccionado por Empresas Promotoras de Servicios de Salud (EPS) e Instituciones Prestadoras de Servicios de Salud (IPS), entre las cuales se presentan relaciones de mercado basadas en una competencia regulada por directrices del Estado ${ }^{1}$.

Desde el inicio de la reforma, la cobertura y el acceso a servicios de salud de calidad fueron condicionados por una brecha económica que marcaba la diferencia entre usuarios con poca capacidad adquisitiva y sin condiciones de empleo formales, que los vinculaba a un denominado régimen subsidiado, en el que el plan de beneficios en salud era limitado y con grandes barreras de accesibilidad. Contrario a esto, aquellos usuarios con condiciones económicas y de empleabilidad más estables tenían acceso a una oferta más amplia tanto de servicios como de prestadores, aunque igualmente presentaban problemas en la calidad y oportunidad del servicio.

Aunque con la puesta en marcha del actual sistema, uno de los problemas que pretendía resolverse era la baja cobertura, que si bien en términos de afiliación y luego de 20 años ha logrado alcanzarse, no ha resuelto las fallas en la garantía del acceso adecuado a los servicios ni la reducción de la desigualdad en términos de enfermedades prevenibles que se presentan con mayor frecuencia en el régimen subsidiado ${ }^{2}$.

$\mathrm{Al}$ estructurarse bajo un modelo descentralizado, competir en términos de calidad y cumplir con lineamientos de política internacional y normativa nacional, las instituciones que hacen parte del sistema de salud pusieron en práctica las estrategias de participación social con el fin de acercar a la ciudadanía a la formulación de estrategias y toma de decisiones de interés

\footnotetext{
Para mayor comprensión de la estructura inicial del sistema, véase la ley 100 DE 1993.

2 Ver informe de ACEMI (2017) en donde se contrastan condiciones de morbilidad entre ambos regímenes de salud. El informe evidencia cómo ciertas causas de morbilidad prevenibles son más frecuentes en el régimen subsidiado y son relacionadas al contexto social y económico en el que habitan las personas.
} 
colectivo, con el agravante de las tensiones que se generan del interés público versus el privado y que será abordado más adelante en este texto.

\section{Antecedentes de la participación en salud}

La participación ciudadana en el sector salud ha estado estrechamente relacionada con la participación social general, pero presentando sus connotaciones particulares. En la década de los setenta fue incorporada internacionalmente la participación comunitaria en salud como resultado de políticas implementadas por la Organización Mundial de la Salud (OMS), la Organización Panamericana de la Salud (OPS), y agencias internacionales de cooperación que brindaron acompañamiento y control de programas de salud cuya estrategia fundamental era la participación de los usuarios en la prestación de los servicios (Álvarez \& Vahos, 1996)

En la conferencia internacional sobre Atención Primaria en Salud (APS), realizada en 1978, se promulgó la declaración de Alma Ata en la que se estableció la APS como estrategia para alcanzar la meta de salud para todos en el año 2000. Desde aquí, la participación comunitaria para la planeación de la salud se convirtió en derecho y deber de los pueblos para favorecer el desarrollo social y económico (Organización Mundial de la Salud, 1978).

Posteriormente, la carta de Ottawa, como resultado de la primera conferencia internacional de atención primaria en salud realizada el 21 de noviembre de 1986, recalca la importancia de la participación comunitaria para el alcance de la meta de salud para todos en el año 2000 y de forma textual cita lo siguiente:

La promoción de la salud radica en la participación efectiva y concreta de la comunidad en la fijación de prioridades, la toma de decisiones y la elaboración y puesta en marcha de estrategias de planificación para alcanzar un mejor nivel de salud. La fuerza motriz de este proceso proviene del poder real de las comunidades, de la posesión y del control que tengan sobre sus propios empeños y destinos. El desarrollo de la comunidad se basa en los recursos humanos y materiales con que cuenta la comunidad misma para estimular la independencia y el apoyo 
social, así como para desarrollar sistemas flexibles que refuercen la participación pública y el control de las cuestiones sanitarias. Esto requiere un total y constante acceso a la información y a la instrucción sanitaria, así como a la ayuda financiera (Organización Mundial de la Salud, 1986, p. 3).

En Colombia, a finales de la década de los ochenta y principios de los noventa, con el objeto de abrir canales de participación ciudadana, el Ministerio de Salud expidió los decretos 1216 y 1416 mediante los cuales se crearon y reglamentaron los comités de participación comunitaria (COPACOS) que le permitía a la población apoyar la planeación y ejecución de los programas de salud y fortalecer el enfoque de descentralización de las reformas constitucionales de la época. Así mismo, la reforma al sistema de salud, mediante la Ley 100 de 1993, erige la participación como uno de los principios orientadores del sistema (Congreso de Colombia, 1993).

Posteriormente, mediante el decreto 1757 de 1994, surgen en Colombia las asociaciones de usuarios que les permiten a todas las personas afiliadas al Sistema General de Seguridad Social en Salud participar de manera activa en la planeación y control de las instituciones del sistema de orden público, privado o mixto (Congreso de Colombia, 1994).

Actualmente, la nueva Ley Estatutaria en Salud 1751 de 2015, en su artículo 12 , establece que "el derecho fundamental a la salud comprende el derecho de las personas a participar en las decisiones adoptadas por los agentes del sistema de salud que la afectan o interesan" (Congreso de Colombia, 2015); por lo anterior, es importante entender que las decisiones en salud socialmente deben considerar a sus involucrados directos garantizándoles el derecho de ser partícipes de ellas.

\section{Aspectos reflexivos de la participación en el contexto colombiano}

Siguiendo el concepto textual de la Organización Panamericana de la Salud sobre participación social en Salud, esta lo define como: 
La acción de actores sociales con capacidad, habilidad y oportunidad para identificar problemas, necesidades, definir prioridades, y formular y negociar sus propuestas en la perspectiva del desarrollo de la salud. La participación comprende las acciones colectivas mediante las cuales la población enfrenta los retos de la realidad, identifica y analiza sus problemas, formula y negocia propuestas y satisface las necesidades en materia de salud, de una manera deliberada, democrática y concertada (Organización Panamericana de la Salud, 1994, citada por Restrepo y Málaga, 2004, p. 204).

De acuerdo con esta definición, la importancia de la participación en las instituciones de salud radica en que los sujetos involucrados tengan capacidad de acción, diseño y ejecución de estrategias de salud propias que promuevan el pluralismo y las visiones propias en el proceso saludenfermedad. La articulación entre desarrollo y salud solo es posible mediante el pronunciamiento dinámico y abierto de todos los involucrados. Como lo expone Ruiz et al. (2016), la idea no es orientar a la gente en el cumplimiento de ideales formulados por agencias internacionales ajenas a su realidad local, sino de construir alternativas desde la cosmovisión de los sujetos y las comunidades, de manera que permita horizontes interculturales diversos para el alcance de las distintas visiones de desarrollo (p. 384).

Entender la salud como un asunto comunitario, no solo aporta herramientas más amplias para su comprensión, sino que también ofrece enfoques más allá de lo clínico y asistencial, en donde lo público cobra valor y las estrategias de atención se tornan más efectivas porque reducen las brechas de acceso a los servicios médicos.

A pesar de los giros y dinámicas que debe asumir la institucionalidad en Colombia para una adecuada participación social, coexisten elementos de fondo que impiden un correcto engranaje entre las instituciones y la ciudadanía, y que a la larga han generado condiciones de exclusión y desigualdades sociales manteniendo imperante el interés privado y las visiones externas sobre la forma como se ejecutan los programas de salud. El primer elemento por considerar es el modelo económico: 
Como lo describe Evans (2007), bajo el concepto de monocultivo institucional, Colombia asume para el sistema de salud un modelo económico de corte neoliberal ajeno a su realidad interna (pp. 23-24, 279); si bien Estados Unidos es el sugerente de dicha estrategia, el modelo de Estado liberal de ese país y sus condiciones económicas están mejor articuladas con la estrategia propuesta. En cambio, el interés de Colombia de estructurarse como un Estado Social de Derecho contradice por completo la estructura de un sistema de salud neoliberal que no se articula con las competencias económicas internas.

Además de esto, convertir la salud en una nueva forma de acumulación de capital abre la puerta a toda una serie de tensiones sociales, en donde las decisiones en materia de nuevas acciones de salud y prestación de servicios deslegitima la opinión de los actores sociales al primar las necesidades de lucro y el paradigma biomédico externo. Frente a tal situación, las estrategias de deliberación y participación ciudadana pierden fuerza en el sentido que el sistema democrático corporativo convierte la voz y las necesidades de todo un colectivo de usuarios en una solo opinión, en el contexto de un conjunto de miembros directivos defensores de sus propios intereses. El segundo elemento es la captura del estado, que Luis Jorge Garay (2008) define como:

Una especie de corrupción económica a gran escala en la que agentes privados influyen en la formulación de leyes, normas, decretos, regulaciones y políticas públicas, en la búsqueda de favorecer sus propios intereses egoístas y en detrimento del bienestar general. En este sentido, como las leyes son de aplicación permanente, sus retribuciones podrán ser permanentes y de largo plazo (p. 10).

Partiendo de este concepto es posible cuestionar algunas de las razones que facilitan las fallas del sistema de salud en Colombia. Si bien es difícil demostrar empíricamente las formas en las que las instituciones de salud influyen en la formulación de leyes que favorecen y perpetúan su accionar económico, distintas investigaciones e informes publicados desde diversas disciplinas han demostrado la ineficiencia e inviabilidad del actual modelo. A pesar de esto, las constantes reformas orientadas a resolver los problemas de corrupción, de accesibilidad, y de oportunidad, solo han generado cambios parciales mas no sustanciales que garantizan el actuar de las empresas 
promotoras de salud y la pérdida de recursos que sacrifican buena parte de las condiciones de bienestar social.

Un Estado que priorice el bienestar general sobre el individual sería más abierto a las voces locales, a la multiculturalidad, a las formas de ver los procesos de salud-enfermedad y a garantizar acceso a servicios de salud incluyentes en donde las condiciones económicas de los ciudadanos no sean razón de exclusión ni limitantes para el alcance de libertades que favorezcan el bienestar y el alcance de condiciones de vida dignas. El tercer elemento es el empoderamiento y la gobernanza:

Retomando las ideas de Evans (2007), una institucionalidad exitosa como centro de desarrollo, está complementada por una tensión armónica por parte de una fuerza de oposición (p. 19). En ese sentido, una sociedad civil informada que actúa de forma activa y participativa procura que las fuerzas del gobierno garanticen los intereses colectivos, de manera que la idea local de desarrollo avanza hacia el alcance de sus fines. Por el contrario, una sociedad civil poco informada, que actúa de forma pasiva, es susceptible a decisiones gubernamentales autoritarias que defienden intereses particulares, de manera que las ideas de desarrollo se mueven hacia atrás, convirtiéndose así en un Estado depredador que sin ninguna consideración ética destruye todo lo que encuentra a su paso dejando de lado el bienestar social.

\section{Conclusiones}

A pesar de que las reformas normativas han impulsado un gran número de estrategias de participación social en salud como elementos de deliberación y democracia, el modelo económico del actual sistema de salud es el principal obstáculo para la inclusión de los usuarios en la toma de decisiones y formulación de planes, programas y proyectos de interés colectivo. Esto, debido a que al ser las instituciones de salud un elemento de acumulación de capital, los intereses privados y las necesidades de lucro priman sobre la garantía del bienestar general.

Mientras las dinámicas del Estado se muevan a favor de los grandes grupos económicos, y los recursos para inversión social tengan como principal 
destino intermediarios particulares, las posibilidades de que las personas sean partícipes de su propio desarrollo se ven limitadas en la medida que los actores involucrados no generen fuerzas de resistencia activa y empoderamiento por la defensa de los intereses colectivos y la inclusión en la formulación de estrategias orientadas a mejorar el bienestar social.

\section{Referencias}

ACEMI. (2017). Cifras e indicadores del sistema de salud. https://www.acemi.org.co/images/ publicaciones/documentos_de_interes/documentos-interes/Informe_Cifras_2017.pdf

Álvarez, L., \& Vahos, J. (1996). Comités de Participación Comunitaria en Salud ¿Espacios para el ejercicio de la democracia? Universidad de Antioquia.

Congreso de Colombia. (1994, 3 de agosto). Decreto 1757. Por el cual se organizan y se establecen las modalidades y formas de participación social en la prestación de servicios de salud. Diario Oficial 41477. https://www.defensoria.gov.co/public/Normograma\%20 2013_html/Normas/Decreto_1757_1994.pdf

Congreso de Colombia. (2015, 16 de febrero). Ley 1751. Por medio del cual se regula el derecho fundamental a la salud y se dictan otras disposiciones. Diario Oficial 49427. http:// secretariasenado.gov.co/senado/basedoc/ley_1751_2015.html

Evans, P. (2007).Instituciones y desarrollo en la era de la globalización neoliberal. Ilsa. http://www2. cmq.edu.mx/libreria/index.php/582/11/sociedad-civil/instituciones-y-desarrollo-detail

Garay, L. J., Salcedo Albarán, E., de León-Beltrán, I., \& Guerrero, B. (2008). La Captura y Reconfiguración Cooptada del Estado en Colombia. Fundación Método, Fundación Avina y Transparencia por Colombia.

Organización Mundial de la Salud (OMS). (1978, 12 de septiembre). Conferencia internacional de Atención Primaria en Salud. http://www.who.int/topics/primary_health_care/es/

Organización Mundial de la Salud (OMS). (1986, 17 de septiembre). Carta de Ottawa para la Promoción de la Salud. https://www.paho.org/hq/dmdocuments/2013/Carta-de-ottawapara-la-apromocion-de-la-salud-1986-SP.pdf

Organización Panamericana de la Salud (OPS). (1994). Evaluación para el fortalecimiento de procesos de participación social en la promoción y el desarrollo de la salud en los sistemas locales de salud. Washington D.C

Orozco Africano, J.M. (2006). Porqué reformar la reforma. Edición electrónica. www.eumed. net/libros/2006/jmo/ 
Ruiz Lurduy, R., Rocha Buelvas, A., \& Pérez Hernández, Elizabeth Córdoba Sánchez, C. (2016). Desarrollo social y salud pública. Reflexiones en torno a la interculturalidad. Revista Facultad Nacional de Salud Pública, 34(3), 380-388. http://dx.doi.org/10.17533/ udea.rfnsp.v34n3a12

Restrepo, H y Málaga, H. (2002). Promoción de la salud: cómo construir vida saludable. Editorial Médica panamericana. 